\title{
A STUDY OF THE EFFECT OF STREPTOHYDRAZID ON LEPROMATOUS LEPROSY OVER A PERIOD OF ABOUT THREE YEARS
}

\author{
JoHN DREISBACH, M.D.* \\ R. G. Cochrane, M.D., F.R.C.P.*
}

In view of the fact that Isonicotinic Acid Hydrazid and Streptomycin have been shown to have an effect on the M. leprae, it was considered advisable to test the combination of these two drugs in leprosy. A study was started, therefore, on a group of lepromatous cases in January, 1954. In March, 1956, 4 more cases were added to this series. In all 59 cases have been studied, of which 47 will be included in this report. Those not included had insufficient treatment to be of any significance in this evaluation, but the length of treatment of the remaining 47 ranged from 10 to 40 months with an average of 32 months' treatment. During the period of experimental trial one patient died after 24 months treatment. He had shown an initial clinical improvement but after 18 months he showed a marked regression in symptoms, but in spite of all that could be done, he died some 4 months after Streptohydrazid was discontinued. Apart from this case, all the other patients continued treatment and stood the drug well.

In January, 1956, one of us (R.G.C.) returned to Kano and with the co-author made a preliminary assessment of the patients under trial with Streptohydrazid, and during this assessment it was noted that patients who had previous sulphone therapy appeared to do better than patients who had been placed on Streptohydrazid alone. Therefore, a group of cases was selected and placed on $50 \%$ Sulphetrone solution injected intramuscularly twice a week : 28 cases were placed on additional Sulphetrone by injection and 18 cases remained on Streptohydrazid alone. One case was not included in the continuation of the experiment, making a total of 46 cases. The $50 \%$ solution of Sulphetrone was chosen because in our experience heavy lepromatous cases showed less reaction and improved better under intramuscular injection of $50 \%$ Sulphetrone than with oral DDS, and it has been shown by one of us (R.G.C.), in a previous communication that parenteral Sulphetrone in the form of a $50 \%$ solution does not break down into DDS. The dose of parenteral Sulphetrone which was given was 3 ccs. of a $50 \%$ solution of Sulphetrone, intramuscularly, twice a week; that is, 3 gms. a week.

We record the following general observations. Firstly, only lepromatous cases were included in this study, and before the experiment started all cases had a complete physical examination, with a

- From the Yadakunya Leprosarium, Sudan Interior Mission, Kano, N. Nigeria.

- The Leprosy Research Fund, 11 a Weymouth St., London W.I. 
biopsy, photographs, routine haematology, stool and urine examinations, and skin smears. Routine laboratory studies were repeated monthly, and the biopsies and photographs were re-taken approximately every six months. All cases which showed histologically atypical lepromatous features were excluded from this study, because it is well known that cases of the dimorphous group show a considerable tendency to spontaneous regression and, therefore, all lepromatous cases were confirmed by biopsy examination.

With the possible exception of the patient who died, there were no serious toxic manifestations. There was some evidence of erythema nodosum leprosum reactions. However, more than $50 \%$ had no such reactions during the course of the treatment. The results were as follows :-

$\begin{array}{llllr}\text { No reactions } & \ldots & \ldots & \ldots & 25 \\ \text { One reaction } & \ldots & \ldots & \ldots & 7 \\ \text { Two reactions } & \ldots & \ldots & \ldots & 6 \\ \text { Three reactions } & \ldots & \ldots & \ldots & 4 \\ \text { Multiple reactions } & \ldots & \ldots & \ldots & 5\end{array}$

In view of the fact that all the cases which were chosen were moderate to heavy lepromatous cases we feel that this result is significant. It is our opinion, based on many years experience with the parent sulphone, that had this been used in these cases a much higher rate of erythema nodosum reactions would have been noted. As it was, the great majority of the reactions, which were precipitated during treatment, were of such a mild nature that it was possible to continue treatment without interruption, but in a few cases of severe reaction Cortisone was administered and made possible the continuance of the treatment. In the course of the experiment the patients showed no alteration in their W.B.C. count or in the Differential count, but there was some evidence of anaemia as follows:-

$\begin{array}{lcccr}\text { No evidence of anaemia } & \ldots & \ldots & 18 \\ \text { Slight evidence } & \ldots & \ldots & \ldots & 12 \\ \text { Moderate evidence } \ldots & \ldots & \ldots & 15 \\ \text { Marked evidence } & \ldots & \ldots & \ldots & 2\end{array}$

All except those with marked anaemia were continued on treatment. Inorganic iron $\left(\mathrm{FeSO}_{4}\right)$ was added and the patients responded satisfactorily. In those with marked anaemia - that is, with less than 7.5 gms. \% - the drug was stopped and more vigorous anti-anaemic measures were instituted. All were later continued in the study.

No toxic manifestations such as drug rash, hepatitis, renal impairment, dermatitis, or central nervous system complications were encountered.

\section{General Conclusions}

Most of the improvement was seen in the first 6 to 12 months. Those cases put on Sulphetrone continued to show further improve- 
ment. The most noteworthy point, however, was that the mucosa of lesions healed very rapidly. Nasal septal ulcerations and nodulations, buccal, labial, lingual, and pharyngeal nodulations seemed to heal quickly-more rapidly than we would have expected with patients on sulphone therapy. In some cases healing was first noted one month after treatment began. Patients with laryngeal symptoms also improved greatly, and in two cases we saw this change on direct laryngoscopy.

In a more detailed analysis of these results it is interesting to note that there was some difference between the cases which received Streptohydrazid alone and those cases in which Streptohydrazid was combined with sulphone therapy. In the latter group of cases it did not seem to matter whether sulphone therapy in the form of a $50 \%$ solution of Sulphetrone was given continuously with the Streptohydrazid or intermittently - that is, 3 months Streptohydrazid and 3 months Sulphetrone. The following Table shows the analysis of the results in cases under Streptohydrazid alone and those given Streptohydrazid in combination with Sulphetrone:

\begin{tabular}{c|c|c|c|c}
\hline & Improved & $\begin{array}{c}\text { Much } \\
\text { Improved }\end{array}$ & Neg. & $\begin{array}{c}\text { Period } \\
\text { under } \\
\text { Treatment }\end{array}$ \\
\hline $\begin{array}{c}\text { Streptohydrazid } \\
\text { alone }\end{array}$ & $22 \%$ & $16 \%$ & - & 32 \\
\hline $\begin{array}{c}\text { Streptohydrazid } \\
\text { intermittent } \\
\text { with Sulphetrone }\end{array}$ & $18.5 \%$ & $33.3 \%$ & - & 32 \\
\hline
\end{tabular}

\section{SELECTION OF ILLUSTRATIVE CASES}

The following four cases are selected among the 47 cases chosen for this drug trial as examples of improvement under Streptohydrazid medication. The photographs are by Dr. R. G. Cochrane and the photomicrographs by the Institute of Dermatology.

BETI MALLAMAWA, age 29 (1954), male.-Advanced lepromatous leprosy.

Case No.-Katsina 1251. See Figs. 1 and 2, 3 and 4.

Previously had Hydnocarpus treatment for five years, 1945-1950.

Commenced Streptohydrazid treatment March 1954. Placed on Intermittent Streptohydrazid treatment and continuous sulphetrone (50\% parenterally) January 1956.

Patient at the time showed heavy infiltrations throughout the body and nodular lesions on the ears and face. Also marked nodulation of the tongue giving the appearance of a geographical tongue.

The patient showed marked clinical improvement between March 1954 and August 1954 - and this improvement continued but at slower rate up to December 1956. 


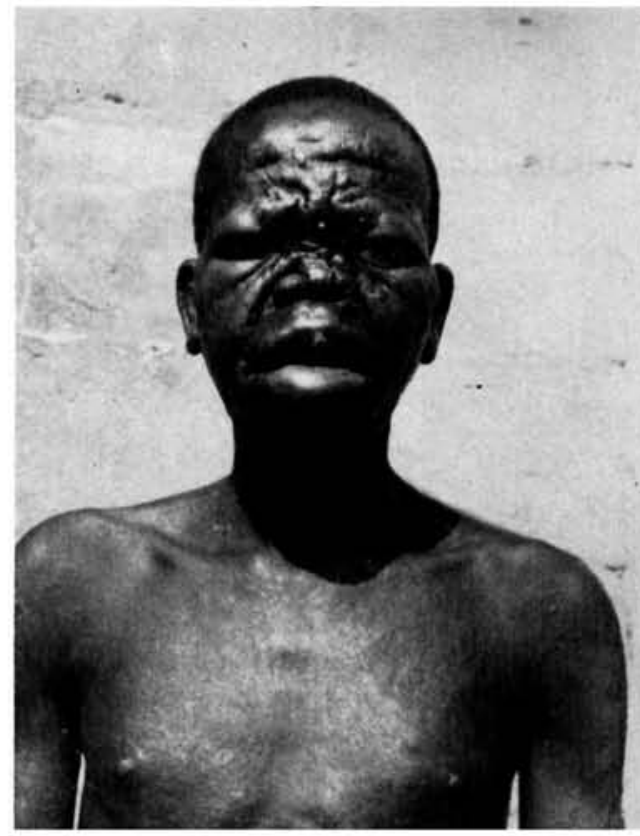

Fig. 1. Beti.

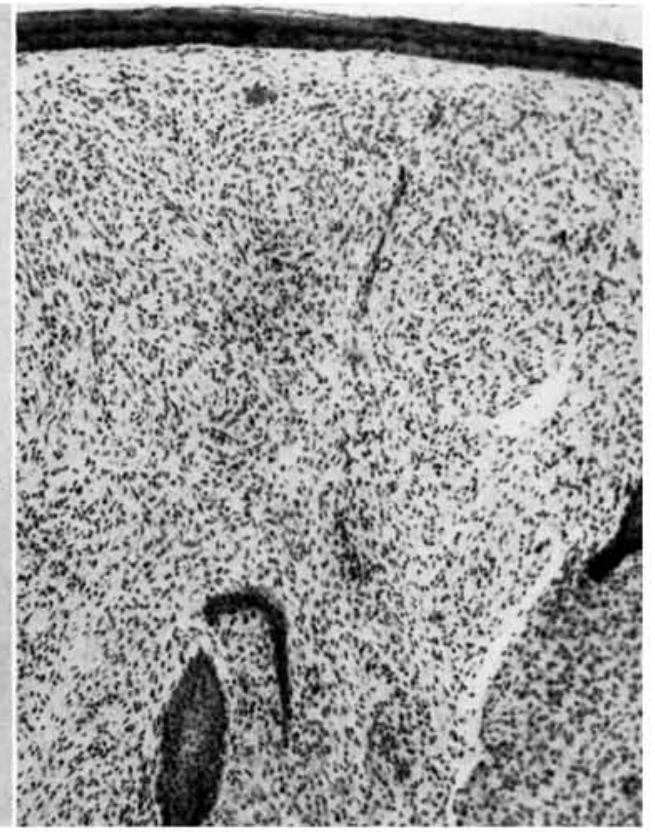

Fig. 2. Beti, before treatment.

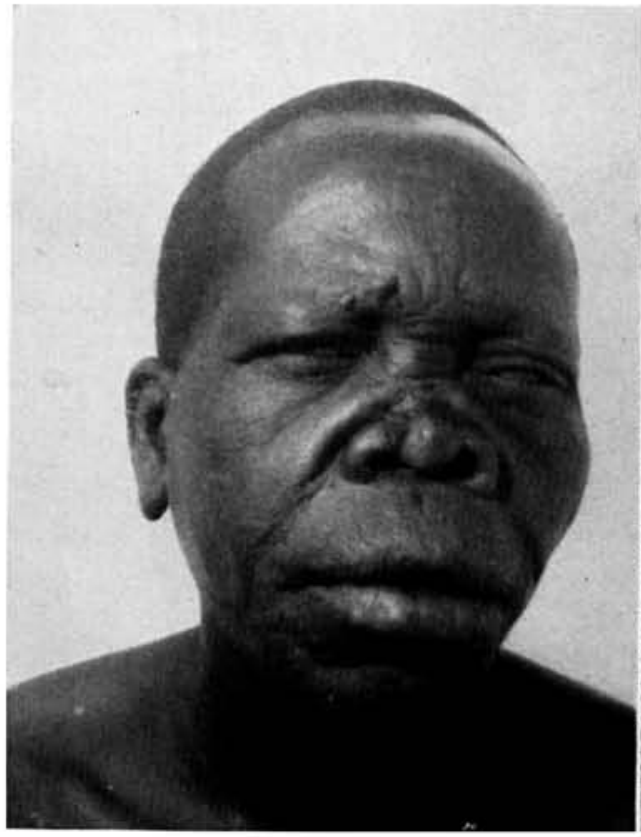

Fig. 3. Beti.

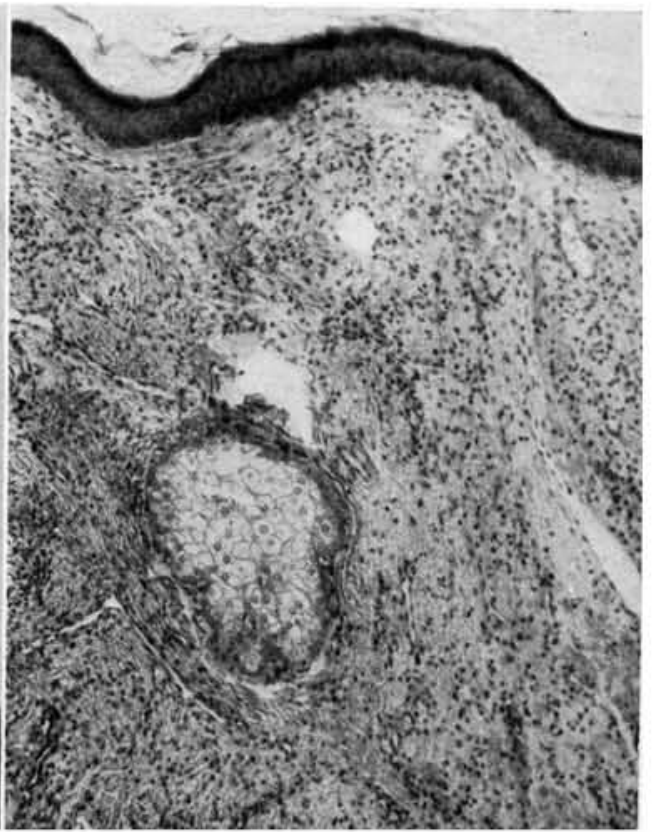

Fig. 4. Beti, after two years' treatment. 


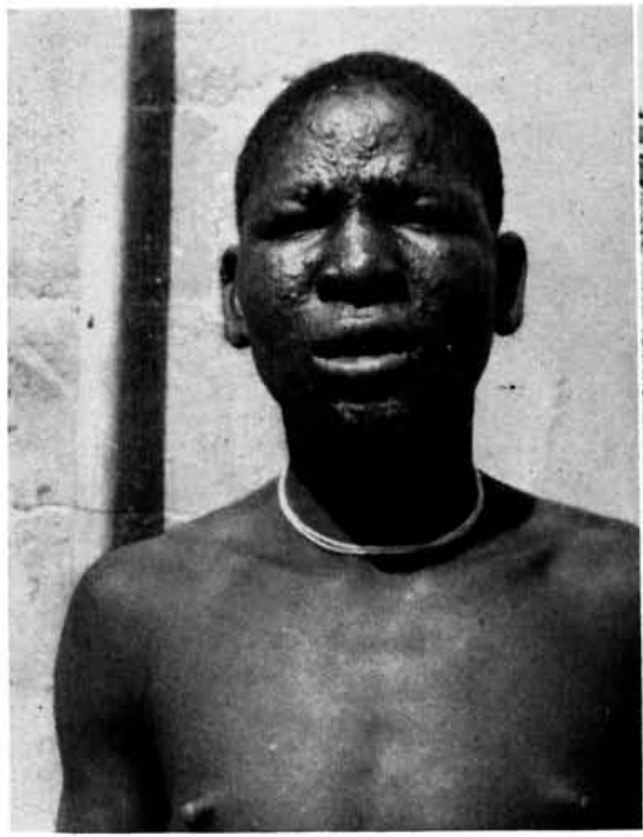

Fig. 5. Usman.

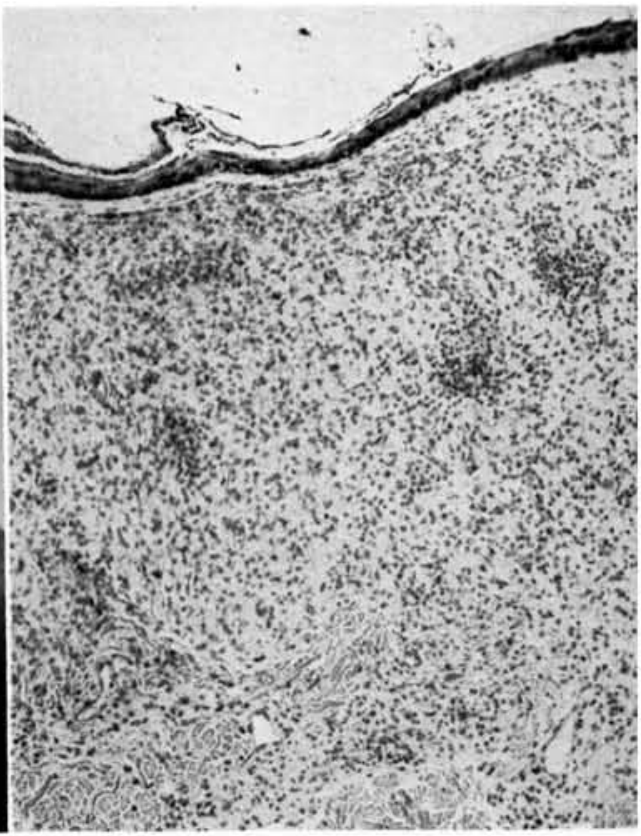

Fig. 6. Usman, before treatment.

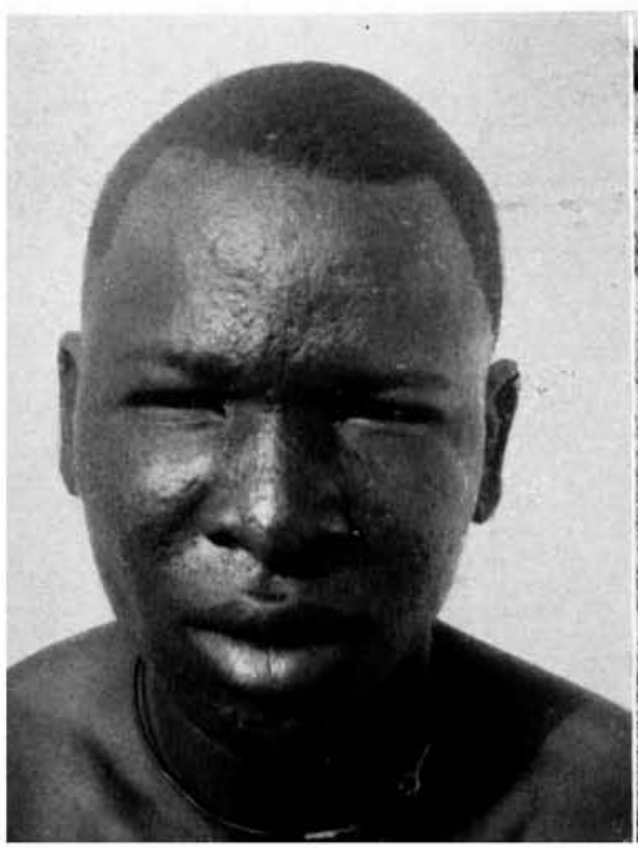

Fig. 7. Usman.

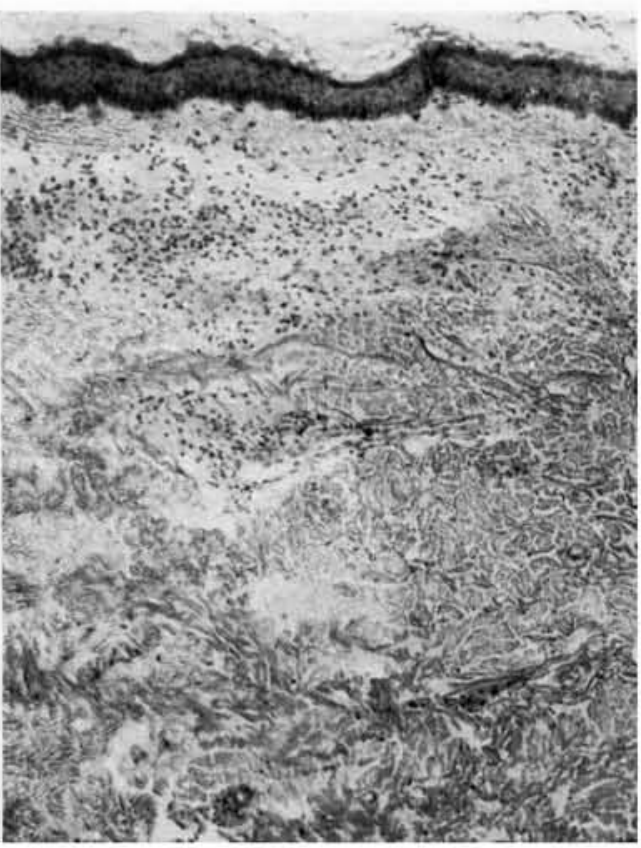

Fig. 8. Usman, after two years' treatment. 


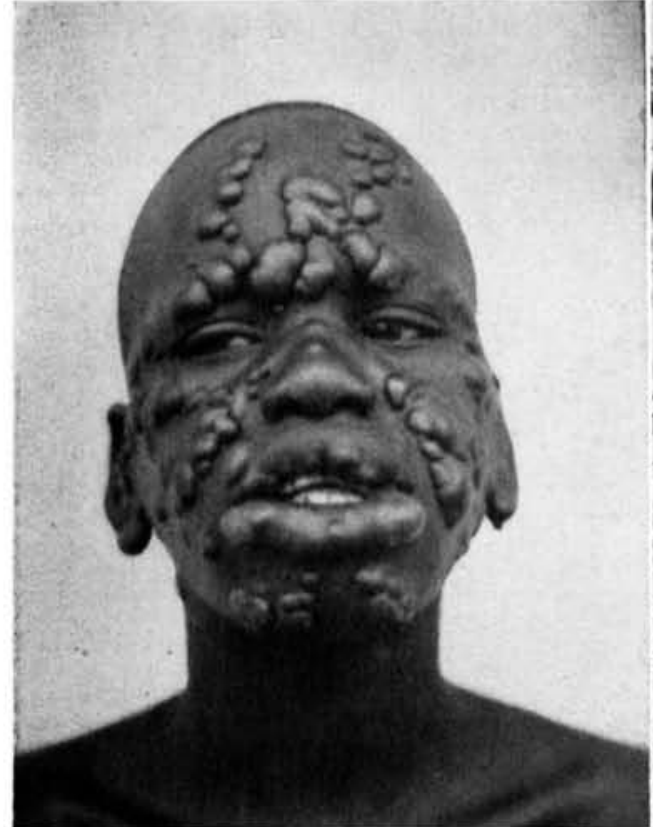

Fig. 9. Gambo.

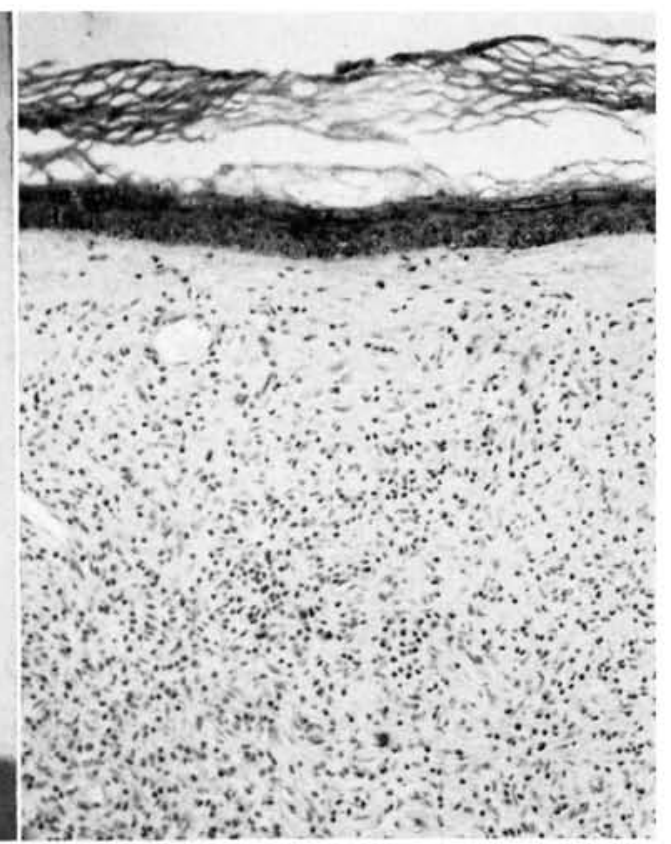

Fig. 10. Gambo.

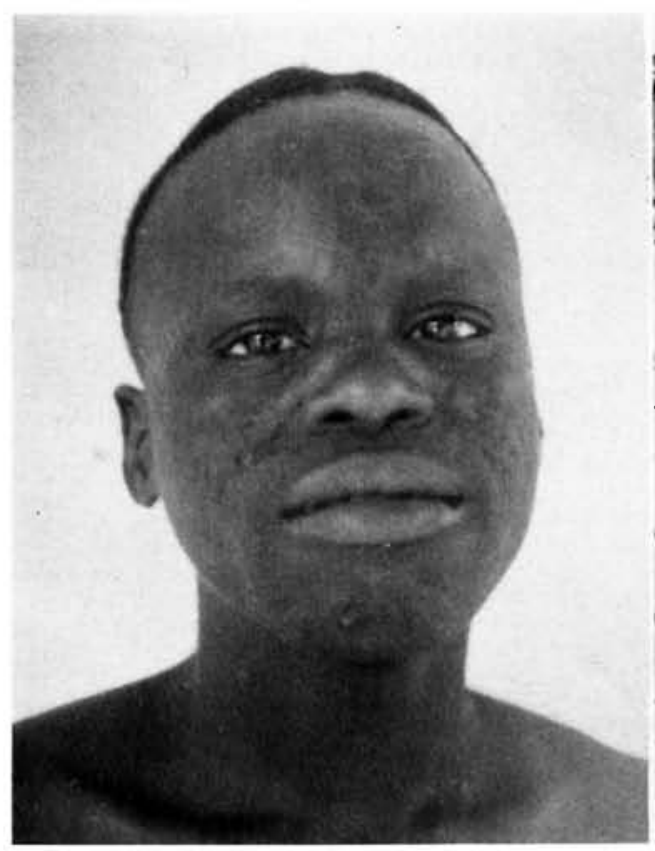

Fig. 11. Gambo.

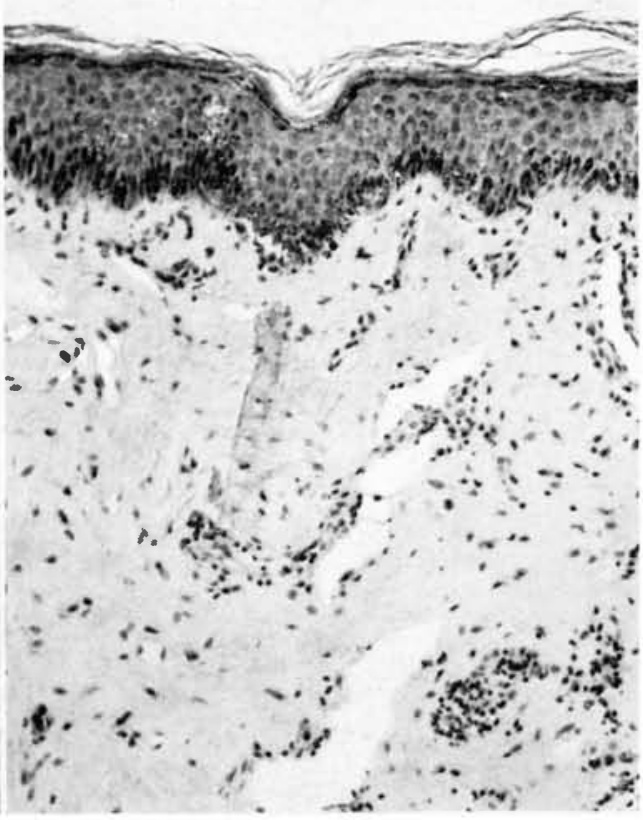

Fig. 12. Gambo. 


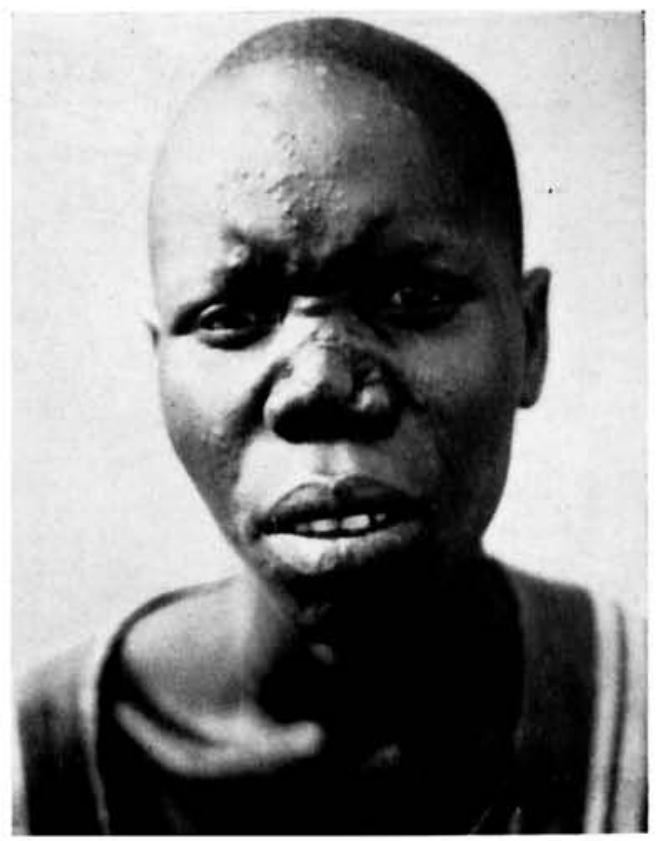

Fig. 13. Kubiya.

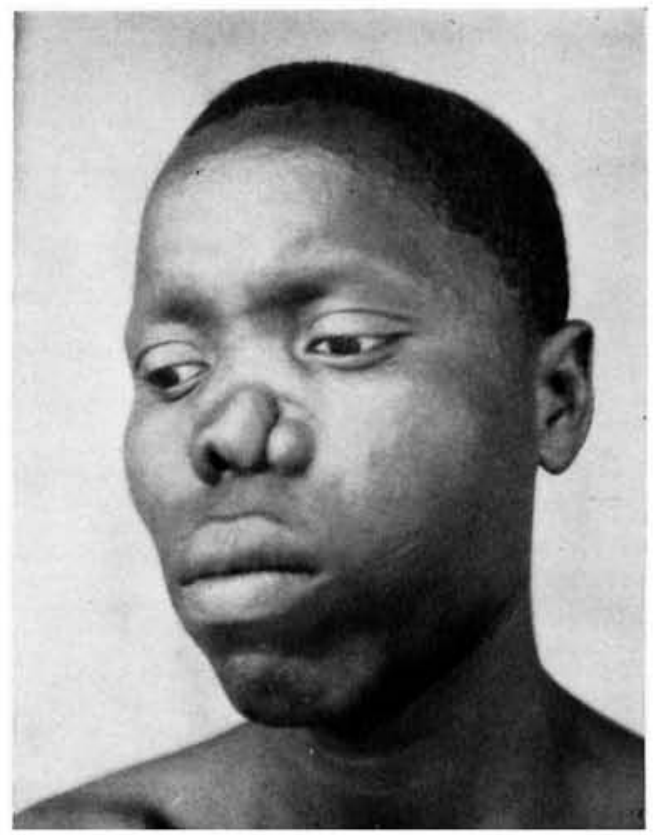

Fig. 14. Kubiya. 
The following are the biopsy reports:

Biopsy' of 27.3.54 (Lab. No. 1332)

The whole of the corium is filled with a massive infiltration leaving a flattened epidermis with a narrow free subepidermal zone. The infiltrate is seen to consist of macrophages which have mostly undergone foamy cell change. There are scattered giant cells but not of the Langhan's type. Many of these also show foamy cell change. Nerves are impossible to distinguish in this mass of infiltrate. Among the foamy cells is a superimposed round cell infiltration. Section stained for acid fast hacilli (Fite Faraco technique)

Numerous acid fast bacilli showing some degree of morphological change. Bacilli are seen in all the macrophage cells including the giant cells.

Biopsy of 23.8.54 (Lab. No. 1532)

This shows a scattered round cell infiltration. The infiltrate is diffuse and in no way focalised. In addition to these round cells there are seen small areas in which there is definite foamy cell change. Nerves are clearly seen and uninvaded. Acid fast section (Fite Faraco technique)

A few granular bacilli are scattered through the section. The bacilli are granular and stumpy looking and are also seen in the nerves.

Comment:

This is a picture of a lepromatous case resolving very satisfactorily under therapy. The residual round cell infiltration suggests some activity of the lesion. Biopsy of 16.4.56 (Lab. No. 2148)

The superficial part of the dermis underneath the epidermis is occupied by a very narrow band of infiltrate with a clear subepidermal zone. The infiltrating cells consist of round cells and histiocytes, and there are also areas of foamy cell change. In the deeper parts of the dermis the infiltrate is very much less. There are one or two small areas of foamy cell change, otherwise there seems to be some increase of collagen but no infiltrate of any significance. Nerves when seen are uninvaded.

Acid fast section (Fite Faraco technique)

The acid fast bacilli appear to be scanty in numbers. They are scattered throughout the foamy cell areas and in the nerves.

Comment: healing.

This is a picture of a resolving leproma which is progressing towards

USMAN GARNI, age 18 (1954), male.

Katsina No. 1545. See Figs. 5, 6, 7, 8.

Previous therapy up to January 1949-Hydnocarpus oil from 1949-1954.

Has been on sulphone therapy but there was no appreciable improvement.

Commenced Streptohydrazid treatment March 1954-placed on Intermittent Streptohydrazid and continuous sulphetrone January 1956.

Between March 1954 and January 1955 considerable clinical improvement.

From January 1955 to December 1956-improvement continued but bacteriological index remained moderately high and only improving from an index of 5 to an index of 4.1 - but the clinical improvement was quite definite. The following are the biopsy reports:

Biopsy of 27.3.54 (Lab. No. 1331)

The section shows all the features of a moderately advanced lepromatous case with gross foamy cell change.

Acid fast section (Fite Faraco technique)

Large numbers of acid fast bacilli throughout the section with numerous globi. The globi show a tendency to break up, and the acid fast bacilli show considerable morphological change.

Biopsy of 16.4.56 (Lab. No. 2159)

The granuloma shows considerable clearing particularly in the deeper areas of the corium. There is some infiltration underneath the epidermis leaving a clear subepidermal zone. The infiltrating cells are chiefly histiocytes and round cells and there is still some evidence of foamy cell change. The nerves are seen and are uninvaded by cellular infiltrate. There is some proliferation of the epineurium.

Acid fast section (Fite Faraco technique) change.

Acid fast bacilli are moderately numerous showing gross morphological

Comment:

This shows satisfactory commencing resolution. 
(GAMBO YARI)AJ, age 15 (1954), malc.-Advanced nodular lepromatous case. Case No. Katsina 2299. See figs. 9, 10, $11,12$.

No previous treatment.

Commenced Streptohydrazid treatment on admission in Junc 1954 but was placed on Intermittent Streptohydrazid and continuous sulphetrone in January 1956.

At the time of admission the patient showed multiple confluent, large firm nodules-particularly on the face and ear lobes, although the whole of the trunk was affected.

Between June 1954 and December 1956-very marked clinical improvement with an almost $30 \%$ improvement in the bacteriological index.

During the course of treatment patient showed no reactions and the progress was maintained.

The following are the biopsy reports:

Biopsy of July 1954 (Lab. No. 1450)

The whole of the corium is occupied by a closely packed mass of granulomatous tissue. The infiltrate consists chiefly of histiocytes and near the epidermis there are several multinucleated cells, there is some evidence of foamy cell change, but this is not conspicuous. Nerves when scen are not invaded, and there is a well-marked vascular free subepidermal zone.

Acid fast section (Fite Faraco technique)

Very large numbers of acid fast bacilli throughout the section, many of which are beaded and darkly stained, but morphological change is no more than to be expected with such a large number of bacilli.

Biopsy of 17.12.56 (Lab. No. 2426)

There is scattered round cell and histiocytic infiltration of slight intensity diffusely distributed throughout the upper parts of the corium and extending up towards the epidermis, but there is evidence of a clear sub-epidermal zone. Cellular infiltrate consists chiefly of histiocytes and round cells. There is no significant evidence of foamy cell change. Again, the section is a poor one for nerves, but one nerve is completely uninvolved by infiltrate.

Acid fast section (Fite Faraco technique)

Very few acid-fast bacilli seen on careful search. An occasional granular bacillus is recognisable.

Comment: therapy.

This is a lepromatous case which has responded extremely well under Biopsy of 3.6.57 (Lab. No. 2761)

The granulomatous infiltration has very appreciably decreased. There is only slight to moderate infiltration underneath the epidermis leaving a clear subepidermal zone. In the deeper parts of the dermis the granuloma has almost completely cleared. The infiltrating cells are chiefly histiocytes, some round cells and an occasional plasma cell. Nerves in the dermis are well seen and are uninvaded and many show marked proliferation of the epineurium. There is some evidence of foamy cell change although this is not marked.

Acid fast section (Fite Faraco technique)

There are acid fast bacilli throughout the section but these are very scanty in numbers. They are seen in the histiocytes and in the nerves. Acid fast bacilli in the nerves show no morphological change.

Comment:

This shows marked resolution of the lepromatous infiltration.

KUBIYA GAYA, age 20 (1954), male. Kano-4186. See Figs. 13 and 14.

No previous treatment recorded.

Commenced Streptohydrazid on May 10, 1954, and placed on Intermittent Streptohydrazid and parenteral Sulphetrone January 1956.

The patient is a well-developed, well-nourished male with definite nodular lesions on the ear lobes and face. Nasal septum ulceration and partial collapse, with partial blocking of the airway. Moderate buccal labial and mild laryngeal involvement. The lesions on the trunk are elevated erythematous plaques with discrete sloping margins and show considerable wrinkling. Lesions on the face are definitely lepromatous and those on the trunk are suggestive of dimorphous leprosy.

From May 1954 to January 1955 the clinical improvement was slight-but from January 1955 to December 1956 the improvement was more marked.

In November 1955 there was histological evidence of a reaction and in January 1956 there was a mild lepra reaction following a tuberculin test. 


\section{Biopsy of 8.7.54 (Lab. No. 1417)}

The whole of the corium is occupied by massive granulomatous infiltration which extends deeply into the dermis. There is very clear free subepidermal zone. The granuloma consists almost entirely of large macrophages many of which show foamy cell change. Here and there are some plasma cells but these do not show significant increase. Nerves when seen are uninvaded with some proliferation of the epineurium.

Acid fast section (Fite Faraco technique)

Large numbers of acid fast bacilli throughout the section with some globus formation. The bacilli show no morphological change.

Biopsy of 14.11.55 (Lab. No. 1955)

The general overall picture is that of a moderate leproma. There is scattered infiltration throughout the corium although this is not seen in a continuous band as is of ten the case in advanced lepromatous leprosy. The subepidermal zone is clear and vascular but amidst this general lepromatous infiltrate which is only moderate in intensity, there is a superimposed more acute inflammatory process, and in some areas this is intense for there are very numerous round cells, the majority of which are polymorpho-nuclear leukocytes. There is also an increase in plasma cells. There is considerable vascularity throughout the section but there are no marked pathological changes in the vessels. Nerves when seen are uninvaded and have the appearance of lepromatous leprosy.

Acid fast section (Fite Faraco technique)

Numerous acid fast bacilli showing morphological change in the areas which are not affected by the acute inflammatory response. In these latter areas the bacilli are very scanty and are almost absent.

\section{Comment:}

This appears to be an acute process of the nature of erythema nodosum superimposed upon moderately advanced lepromatous leprosy. Biopsy of 17.12.56 (Lab. No. 2395)

This shows a very considerable decrease of the granulomatous infiltration which is only slight to moderate in intensity and chiefly in the upper parts of the dermis near to the epidermis there is a relatively clear subepidermal zone. The granulomatous infiltration consists almost entirely of foamy cells. There is some round cell infiltration and some increase of plasma cells. Nerves when seen are relatively uninvaded and show some increase in the cells of Schwann. Acid fast section (Fite Faraco technique)

The number of acid fast bacilli appear to be considerably reduced but they are still seen in significant numbers and the interesting feature is that in the epidermis lining the hair follicles there are masses of acid fast bacilli in among the epidermal cells. Nerves also show numerous acid fast bacilli. Comment:

This is a lepromatous case which is responding satisfactorily to therapy. The granuloma is beginning to clear but the numbers of acid fast bacilli although reduced, still seen in appreciable quantities.

In view of this result it would indicate that there is a more rapid improvement on a combination of sulphone therapy and Streptohydrazid than with either alone.

One of the interesting points to which we would like to draw attention is the very marked improvement in nasal and buccal lesions. It is further interesting to note that 3 cases of clinically diagnosed pulmonary tuberculosis were also included in this study. These all had advanced lepromatous leprosy as well. No X-ray examination or culture of the bacilli was possible, but the diagnosis was made on clinical findings and on positive sputum. The acid-fast bacilli could possibly have been $M$. leprae coughed up from the larynx, but the pulmonary condition cleared with the first year of treatment and the sputum became negative, whereas the skin continued to be positive to $M$. leprae. None now show clinical evidence of tuberculosis. 


\section{Conclusions}

We conclude by this experiment that Streptohydrazid in a dosage of 5.6 gms. per week, given preferably in combination with sulphone therapy, is a method of treatment which we can advocate for the rapid clearing of nasal and buccal lesions, and for those cases which show intolerance to sulphone therapy. When this intolerance is very great we would recommend that Streptohydrazid be given alone for the first year, and then Streptohydrazid and parenteral Sulphetrone be given for the second year. As a result of the above conclusions we have chosen cases for further clinical trial to see whether finally they will become negative to all routine tests more rapidly than similar cases on the parent sulphone or on Sulphetrone when given alone.

\section{Acknowledgments}

We would thank most sincerely Messrs. Chas. Pfizer \& Co., for their generosity not only in supplying the Streptohydrazid for this clinical evaluation but also Cortisone, and our thanks are especially due to Dr. Gladys Hobby for advice and assistance throughout the experiment. 